\title{
Paiement pour services environnementaux (PSE) et motivations des usagers des terres d'un bassin versant. Cas de l'hydroélectricité à Tolongoina, Madagascar
}

\author{
Fano Andriamahefazafy ${ }^{1, *}$, Albert Rakotonirina ${ }^{2}$ et Georges Serpantié ${ }^{3}$ \\ ${ }^{1}$ C3ED-M, Mention Economie, Université d'Antananarivo, BP 905, Antananarivo, Madagascar \\ 2 GRET, Lot II A 119 S Soavimbahoaka, 101 Antananarivo, Madagascar \\ ${ }^{3}$ Institut de recherche pour le développement (IRD), UMR GRED (UPVM-IRD), BP 182, Ouagadougou, Burkina Faso
}

\begin{abstract}
Résumé - Un dispositif de paiement pour services environnementaux (PSE) s'appuie sur l'incitation économique directe pour influencer les comportements pro-environnementaux. Nous nous intéressons dans cet article aux facteurs explicatifs des attitudes des usagers des sols impliqués dans un PSE de bassin versant lié à l'hydro-électrification rurale à Madagascar. En effet, malgré des compensations prévisionnelles et effectives largement inférieures aux coûts d'opportunité induits par des restrictions de pratiques agricoles, ainsi que des dysfonctionnements institutionnels en leur défaveur, ces usagers des sols ont signé un contrat PSE de trois ans en 2013 et respecté leurs engagements écologiques et agricoles durant cette période. À travers une approche qualitative, nous montrons que l'interaction entre motivations intrinsèque et extrinsèque liées à l'espérance de meilleures opportunités à venir, aux bénéfices non monétaires et à des considérations sociales et foncières, a comblé la faiblesse de l'incitation économique directe.
\end{abstract}

Mots clés : Paiement pour services environnementaux (PSE) / incitations / Madagascar / coûts d'opportunité / énergie hydroélectrique

\begin{abstract}
Payment for environmental services (PES) and watershed land user motivations. Case of hydroelectricity in Tolongoina, Madagascar. A payment for environmental services (PES) scheme is based on direct economic incentives to influence pro-environmental behavior. This paper focuses on the factors explaining the attitudes of land users involved in a watershed PES linked to rural hydroelectrification in Madagascar. Indeed, in spite of planned and actual compensations well below the opportunity costs induced by restrictions of agricultural practices, as well as institutional dysfunctions to their disadvantage, these land users signed a three-year PES contract in 2013 and respected their ecological and agricultural commitments during this period. Through a qualitative approach, we show that the interaction between intrinsic and extrinsic motivations related to the expectation of better future opportunities, non-monetary benefits, and social and land considerations, have made up for the weakness of direct economic incentives.
\end{abstract}

Keywords: Payments for environmental services (PES) / incentives / Madagascar / opportunity costs / hydroelectric power

\section{Introduction}

Si les «services écosystémiques» ou «services écologiques » sont considérés comme les services rendus aux hommes par les écosystèmes, les «services environnementaux»

\footnotetext{
$\overline{\text { *Auteur de correspondance }}$ :

fano. andriamahefazafy@gmail. com
}

renvoient à une approche en termes d'externalités positives entre acteurs économiques (Méral, 2012): des attitudes bénéfiques pour l'environnement profitent à d'autres entités qui vont bénéficier de la disponibilité de «services écosystémiques».

La non-prise en compte des externalités positives est une des explications des comportements dégradant l'environnement et de la rareté des actions de protection de ce dernier. Apparus au début des années 2000, les dispositifs de Paiement 
pour services environnementaux (PSE) visent précisément à compenser cette absence par l'internalisation de ces externalités (Karsenty, 2019). Ils introduisent un lien direct, d'un côté, entre le versement d'une contribution de la part des entités qui bénéficient de ces externalités («bénéficiaires » de services environnementaux), et de l'autre, un respect des engagements par les entités qui acceptent, collectivement ou individuellement, d'adopter ou de maintenir des pratiques favorables à l'environnement («fournisseurs» de services environnementaux).

S'appuyant sur ce schéma théorique, une des définitions de référence considère un dispositif de PSE comme «une transaction volontaire où un service environnemental bien défini, ou un usage pouvant assurer la fourniture de ce service environnemental, est "acheté» par (au moins) un client de service environnemental à (au moins) un fournisseur, si et seulement si le fournisseur de service environnemental assure la fourniture ininterrompue de ce service (conditionnalité) 》 (Wunder, 2005). Toutefois, l'évolution des PSE dans le monde depuis vingt ans montre une multitude de dispositifs s'écartant plus ou moins de la considération théorique initiale (ShapiroGarza et al., 2020), ainsi qu'une diversification des définitions, notamment celles basées sur la prise en compte de la diversité des contextes et des arrangements institutionnels (Froger et al., 2016).

Leur caractère basé sur l'incitation économique distingue les PSE des dispositifs de gestion communautaire, tandis que l'aspect direct les distingue d'autres dispositifs indirects tels que les projets de conservation et de développement intégrés (PCDI) (Ferraro et Kiss, 2002). L'incitation économique issue des contributions des bénéficiaires de services environnementaux est considérée comme une compensation des coûts directs (liés à des activités à entreprendre) et/ou indirects (renoncement à des revenus courants ou potentiels, ou «coûts d'opportunité ») engendrés par l'adoption de comportements favorables par les fournisseurs de services environnementaux. Pour être justifié économiquement et être une vraie source de motivation pour les entités concernées, le montant du PSE devrait dépasser les coûts supportés par les fournisseurs de services environnementaux, et être inférieur aux coûts potentiels que les bénéficiaires de services environnementaux ont à subir en l'absence de comportement adapté (Wunder, 2005).

À Madagascar, les dispositifs de PSE ont commencé à rejoindre l'arsenal des outils de gestion de l'environnement à la fin de la première décennie des années 2000 (Bidaud et al., 2013). C'est le cas d'un dispositif pionnier de PSE que l'Organisation non gouvernementale française GRET a initié en 2009 sur un des sites du projet d'hydro-électrification rurale dénommé RHYVIERE I (2007-2015), financé principalement par l'Union européenne (Toillier, 2009). Il s'agit du bassinversant en partie forestier d'Andasy, de la commune rurale de Tolongoina, située en lisière de l'aire protégée du corridor forestier reliant les parcs nationaux de Ranomafana et Andringitra, sur la «falaise» de la côte est de Madagascar. Il s'étend sur 630 hectares, avec une partie agricole de 100 hectares cultivée par une trentaine de ménages. Si les villages riverains du bassin-versant sont peuplés principalement par des autochtones issus de l'ethnie Tanala, les ménages usagers du bassin-versant sont des descendants de migrants de l'ethnie Betsileo (Fig. 1).
Les activités agricoles menées à proximité du futur barrage alimentant la microcentrale étaient perçues par le projet et la mairie de Tolongoina comme menaçant la ressource en eau en termes de qualité (transports solides) et de quantité (niveau d'étiage). Dès 2007, en vue de faciliter la mise en place de ce projet d'électrification, un arrêté communal anti-feu a été mis en place dans le bassin-versant en vue de préserver son couvert végétal. Or, les règles n'ont été que partiellement suivies et des opérations de défriche-brûlis sur de la forêt mature ont même été constatées, entraînant les sanctions prévues à l'encontre des auteurs. Le contexte de l'époque était alors marqué par de vives tensions dans l'application de cet arrêté.

Comme le financement de l'Union européenne ne prévoyait pas de volet PSE, sa mise en place s'est faite à l'initiative du GRET. Pour ce dernier, le PSE était censé calmer les tensions, en accompagnant les ménages usagers de la terre en amont dans l'adoption et le respect de certaines pratiques prévues dans un schéma d'aménagement co-défini entre 2009 et 2012 (pas de travail de sol sur les fortes pentes ou près des ruisseaux, modes de cultures antiérosifs, arrêt des feux et des nouveaux défrichements) (Serpantié et al., à paraître). En contrepartie de leurs efforts, ces usagers de la terre, considérés comme des fournisseurs de services environnementaux, devaient recevoir des compensations sous forme d'appuis en agroforesterie, techniques antiérosives et micro-projets à leur initiative (petit élevage, matériel végétal...). Ces compensations devaient avoir une valeur équivalente au moins aux coûts d'opportunité correspondant à la «restriction d'usage » induite par l'arrêté communal.

Dans une démarche participative avec les acteurs concernés, il a été décidé que les compensations seraient financées par des bénéficiaires du service environnemental : l'exploitant de la centrale et les usagers de l'électricité (les ménages abonnés et la mairie de Tolongoina).

La conception et la mise en œuvre de ce dispositif ont connu des dysfonctionnements au détriment des fournisseurs de services environnementaux (faible niveau de contributions prévisionnelles de la part des bénéficiaires, contributions effectives réduites de moitié, arrêt des appuis après une seule dotation, tension dans la gouvernance du dispositif). Malgré ces défaillances, les fournisseurs de services environnementaux, regroupés au sein d'une association dénommée TAMIS, ont tout de même signé un contrat collectif et respecté les règles établies pendant les trois années du contrat. Il s'agit du premier contrat de PSE hydroélectricité signé à Madagascar (en vigueur de septembre 2013 à septembre 2016). Cet engagement des usagers des terres du bassin versant interpelle et nous amène à nous poser la question de leurs motivations dans ce PSE.

Les aspects relatifs à la motivation des fournisseurs de services environnementaux sont abordés de différentes manières dans la littérature des PSE et à travers des cas variés dans le monde (Bremer et al., 2014; Lapeyre et al., 2016; Rode et al., 2015). Ces recherches mettent en avant le fait que, dans certains cas, l'incitation économique apportée par un dispositif de PSE n'est pas le seul facteur d'explication de la motivation des fournisseurs de services environnementaux. Des considérations extra-économiques peuvent compléter ou se substituer à la dimension économique. L'objectif de cet article est de tirer des enseignements à partir d'un cas observé à Madagascar et d'enrichir la connaissance sur la 


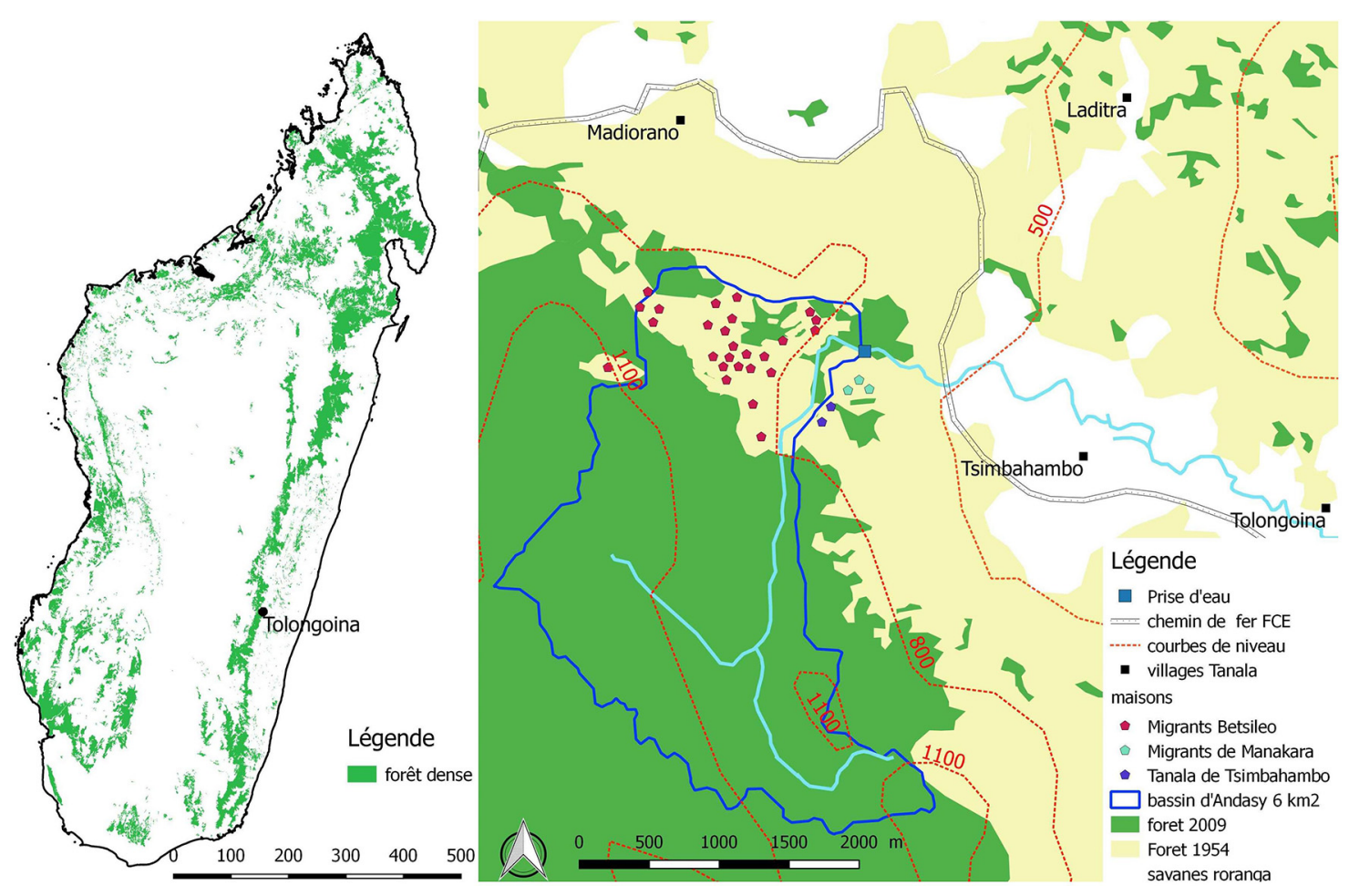

Fig. 1. Evolution de la couverture forestière du bassin versant d'Andasy Tolongoina (source: auteurs et Toillier, 2009).

Fig. 1. Evolution of forest cover in the Tolongoina watershed.

question des motivations des fournisseurs de services environnementaux par rapport au PSE.

\section{Cadre d'analyse et méthodologie}

Le processus de mise en place du PSE s'appuyait sur une recherche-action dans le cadre d'un partenariat entre le GRET et un programme de recherche sur les services environnementaux, dénommé ANR SERENA, porté par une équipe pluridisciplinaire de l'Institut de recherche pour le développement (IRD), du Centre de coopération internationale en recherche agronomique pour le développement (CIRAD) et de l'Institut national de recherche en sciences et technologies pour l'environnement et l'agriculture (IRSTEA), en partenariat à Madagascar avec le Centre d'économie et d'éthique pour l'environnement et le développement à Madagascar (C3EDM). Les co-auteurs de cet article sont impliqués dans ce partenariat.

Si les travaux traitant des motivations autour des PSE mobilisent davantage des approches quantitatives, nous privilégions dans cet article une approche qualitative qui repose sur un croisement de regards entre trois cadres d'analyse: une analyse institutionnelle du dispositif, une analyse socio-économique de la population agricole et une approche agronomique et écologique du système de pratiques agricoles.

L'analyse institutionnelle exploite un matériau varié : des rapports de stage, des notes de réunion, le focus group de l'évaluation de fin de contrat et les observations directes des trois co-auteurs. Les analyses socio-économiques et agronomiques s'appuient sur des enquêtes et des évaluations en début de processus (2009) et en fin de contrat (2016), menées ou encadrées par les co-auteurs (Moyen, 2010; Rakotoarisoa et al., 2016; Ratianarijaona et al., 2016; Andriamahefazafy et al., 2017). La méthode employée pour l'évaluation des impacts écologiques observés pendant la période du contrat écologique consistait en une cartographie au sol (2009) et une cartographie sur image satellite à haute résolution (2015) validée par des observations au sol en 2016 (Rakotoarisoa et al., 2016). Une nouvelle cartographie satellitaire de l'occupation du sol en 2019 a été réalisée par les auteurs à partir d'une image Google Earth.

\section{Engagement des fournisseurs de services environnementaux dans le dispositif de PSE : entre résignation et adaptation}

Nous allons revenir sur le processus de mise en place et sur la phase de mise en œuvre du contrat de PSE, en vue de cerner l'évolution des attitudes des fournisseurs de services environnementaux.

\subsection{Faible contribution des payeurs mais consentement à s'engager des fournisseurs}

La mise en place du PSE par le GRET s'est faite en trois étapes principales entre 2009 et 2013 (Fig. 2).

La première étape a consisté à quantifier et finalement à s'accorder sur les effets comparés de l'agriculture et de la forêt sur le débit d'étiage et la qualité de l'eau (Moyen, 2010; Roche, 2011 ; Serpantié et al., à paraître). 


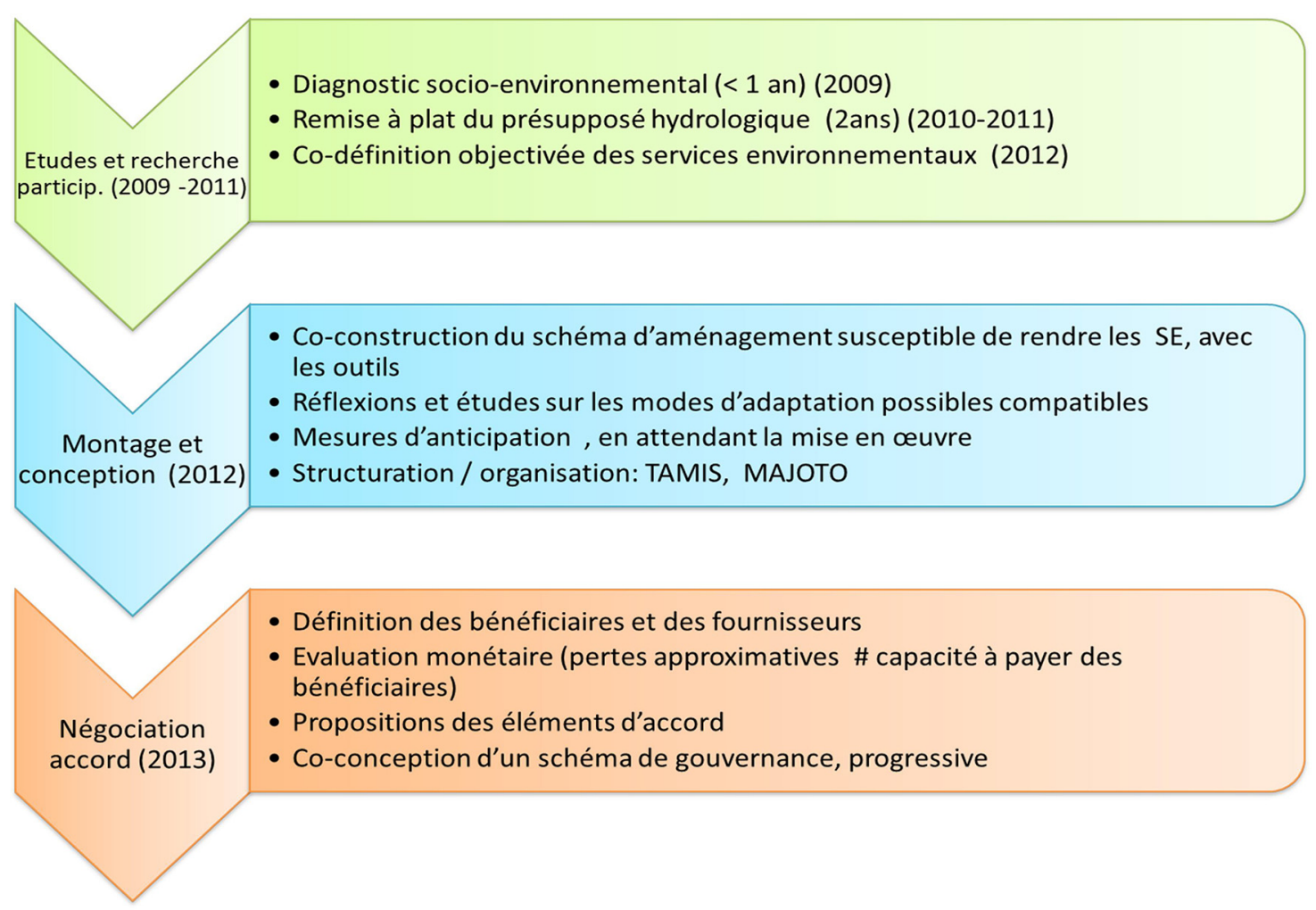

Fig. 2. Les étapes de la mise en place du dispositif PSE (Rakotonirina, 2018).

Fig. 2. The stages of the PES implementation.

La deuxième étape a permis, à travers un processus participatif, de définir les services attendus et d'élaborer un plan d'aménagement précisant les pratiques ou activités à modifier et/ou à encourager, pour une production durable d'hydroélectricité (Serpantié et al., à paraître). Afin d'apporter une compensation aux fournisseurs de services environnementaux, qui ne sont pas parmi les bénéficiaires de l'électrification, le GRET a effectué en 2011 une estimation sommaire des manques à gagner liés aux restrictions sur trois principales cultures vivrières annuelles (riz, manioc et haricot) prévues dans le plan d'aménagement. La culture de riz pluvial (l'alimentation de base) et du haricot (source de revenu secondaire après les bananes) se font toujours avec brûlis, tandis que la culture du manioc se fait avec travail du sol, et, sur les pentes fortes, avec un labour localisé au moment de la plantation, un travail superficiel du sol lors du sarclage, et un travail profond localisé lors de la récolte. Ces cultures devaient soit se faire sans utilisation du feu, soit être abandonnées à partir du lancement du projet d'électrification. Pour les 30 ménages concernés, l'estimation effectuée par le GRET était de $4100 € /$ an (soit une moyenne de $137 € /$ an/ménage) (Rakotonirina, 2018). Il s'agit des revenus agricoles de ces cultures pour le ménage, calculés à partir des produits bruts moins les charges et consommations intermédiaires (semences, main-d'œuvre, amortissement des petits outils de travail). Comme l'ensemble de la production est concerné par les règles d'interdiction, le calcul ne distingue pas entre part autoconsommée et part commercialisée. Par ailleurs, il est à remarquer que l'estimation du GRET s'est basée sur une surface moyenne des parcelles de chaque culture dans le bassin versant et n'est pas entrée dans le détail du revenu total de l'exploitation des ménages.

Dans la troisième étape, afin que le dispositif de PSE puisse générer le montant estimé, différentes réunions ont eu lieu en 2012 et 2013 pour identifier les bénéficiaires à impliquer et les convaincre de contribuer financièrement. Contrairement aux PSE hydroélectricité en général, où le principal payeur est l'entreprise exploitante de l'électricité (ici, il s'agit de la société SM3E), ce PSE se distingue par l'implication de deux autres entités, à savoir la mairie de la commune rurale de Tolongoina (maitre d'ouvrage) et les futurs abonnés de l'électricité (regroupés au sein de l'association MAJOTO).

La modalité de contribution des futurs abonnés a consisté en un prélèvement proportionnel au montant de la facture par compteur, donc variable puisque dépendant du nombre d'abonnés et de leur niveau de consommation. Le taux de $2,5 \%$ a été retenu et validé par consensus par l'ensemble des futurs abonnés. La contribution est estimée à $28 € /$ mois à raison d'une projection de consommation moyenne estimée à $30 \mathrm{kWh} /$ mois pour 450 abonnés.

Malgré la précarité financière des communes rurales à Madagascar, la mairie de Tolongoina a également accepté de contribuer à hauteur de $20 € /$ mois.

Quant à l'entreprise SM3E, si l'ancienne direction avait refusé en 2012 le principe de contribution au PSE, arguant des charges importantes, une nouvelle direction a finalement consenti en 2013, sans se référer à un calcul préalable des coûts qu'elle aurait à subir sans PSE, à contribuer mensuellement à hauteur de $25 € /$ mois. 


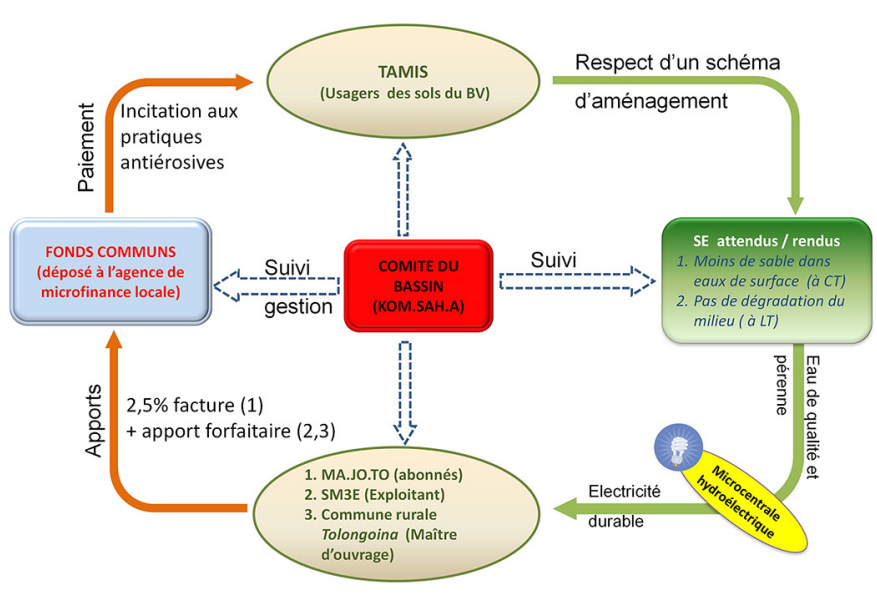

Fig. 3. Schéma du PSE de Tolongoina (Rakotonirina, 2018).

Fig. 3. Tolongoina PES scheme.

Le montant annuel des trois contributions, presque équivalentes, de la part des bénéficiaires de services environnementaux est de $1100 € /$ an, largement inférieur à la somme de $4100 € /$ an issue du calcul des coûts d'opportunité. Bien qu'un tel écart aurait dû rendre la signature du contrat PSE improbable, les fournisseurs de services environnementaux ont tout de même consenti à s'engager.

Le design du dispositif est conforme au cadre logique des PSE en général, avec un schéma que nous pouvons qualifier de «mécanique » (Fig. 3). Une plateforme d'acteurs, formée par des représentants de chaque entité concernée, assure la gouvernance du dispositif. Cette structure, dénommée KOMSAHA (Comité du bassin versant), est censée assurer la supervision de la collecte des contributions provenant des bénéficiaires et la gestion du fonds PSE par rapport aux demandes formulées par l'Association TAMIS. Elle doit également mener des contrôles sur le respect du plan d'aménagement. Les contributions et les retraits se font auprès de la seule institution financière de la localité, une agence de microfinance, mais qui n'est pas signataire du contrat.

\subsection{Dysfonctionnement du paiement mais respect du cahier des charges «fournisseurs"}

\subsubsection{Des dérives dans la mise en œuvre du contrat...}

Le fonctionnement du PSE prévoit que l'association TAMIS devra compiler les demandes d'appui provenant des ménages usagers des sols et formuler trimestriellement une demande de financement d'un microprojet collectif dans le domaine agricole ou de l'élevage auprès de KOMSAHA. Toutefois, une rupture des engagements des bénéficiaires des services environnementaux et une défaillance institutionnelle ont remis en cause cette modalité de fonctionnement.

Tout d'abord, le montant collecté représente moins de $40 \%$ du montant initialement prévu dans le contrat (Tab. 1). Cet écart est dû notamment à la diminution, dès les premiers mois du contrat, des contributions de l'entreprise SM3E et de la mairie. Ces deux entités ont même arrêté de contribuer dès la deuxième année. De plus, les contributions des ménages abonnés n'ont pas atteint le niveau escompté. Si le prélèvement de $2,5 \%$ par facture a permis de disposer d'une contribution systématique et régulière, le niveau de consommation et le nombre des abonnés, avec seulement 250 ménages au lieu des 450 prévus, ont été en deçà des prévisions.

Ensuite, une grande partie du fonds PSE constitué s'est volatilisée à cause de la faillite du réseau régional de l'agence de microfinance au cours de l'année 2014. Bien qu'étant un évènement exogène au dispositif de PSE, cette situation n'est pas sans conséquence sur le fonctionnement du dispositif. Durant les 3 ans de contrat, un seul appui à partir du fonds PSE a pu être délivré aux fournisseurs de services environnementaux en 2014. Il s'agit d'un micro-projet d'élevage avicole d'un montant de $200 €$ pour 10 ménages membres de TAMIS. Entre 2015 et 2016, les contributions des abonnés ont pu continuer à être collectées au sein d'une structure informelle initiée par l'ancienne caissière de l'agence de microfinance. Toutefois, aucun autre micro-projet au profit des usagers des terres n'a pu être financé dans le strict cadre du PSE, alors que la plateforme KOMSAHA a pu retirer assez d'argent sans discontinuer pour assurer ses dépenses de fonctionnement et payer des indemnités de contrôle, certes modiques ( 60 à $70 € / \mathrm{an}$ ).

L'esprit du mécanisme inscrit dans le contrat (privilégier le paiement des fournisseurs du service environnemental) semble donc avoir été oublié, de même que les obligations des autres bénéficiaires, nées de la signature d'un contrat de droit privé, où les contributions au PSE s'apparentent à des «variables d'ajustement».

\subsection{2 ... mais une conformité au plan d'aménagement}

Malgré ce dysfonctionnement dans la mise en œuvre du PSE, l'évaluation menée en 2016 a montré que le plan d'aménagement avait été respecté par les fournisseurs de services environnementaux durant la période du contrat.

L'évaluation cartographique de 2009 à 2016 a fait le constat d'une augmentation légère (de l'ordre de $20 \%$ ) des couverts arborés et arbustifs, aux dépens du couvert herbacé (cultures annuelles, jeunes jachères). Il a même été observé une avancée de la forêt (reforestation) sur le côté sud-ouest du bassin versant agricole, là où des défrichements récents avaient eu lieu et ont été gelés. Cette évolution traduit l'abandon effectif de la pratique du feu. La chute du couvert herbacé traduit aussi un relatif abandon des cultures annuelles ( -21 ha, soit $-70 \%$ ), dont certaines ont été déplacées hors du bassin. Il n'existe pas d'extensions de parcelles par nouvelle défriche de la forêt entre 2009 et 2016: ici encore le schéma d'aménagement a été respecté pendant le contrat (Fig. 4). Cette réduction de la classe des milieux herbacés, qui contiennent les cultures annuelles, entraîne une réduction des risques d'érosion.

Ce constat est renforcé par la situation post-contrat où l'on a pu constater par une cartographie sur image satellite Google Earth de 2019 (donc trois ans après la fin du contrat et en l'absence de nouveau contrat) une forte reprise des milieux herbacés, fortement réduits entre 2009 et 2016 . Un nouveau défrichement est même observable au Sud (Fig. 4). Cette situation confirme l'importance accordée par les fournisseurs de services environnementaux au respect de leurs engagements durant la période du contrat, malgré les imperfections à leur désavantage. 
Tableau 1. Contributions effectives au contrat PSE de 2014 à 2016.

Table. Actual contribution to PES from 2014 to 2016.

\begin{tabular}{lccc}
\hline Montant des contributions de janvier 2014 à mai 2016 & Montant escompté dans le contrat $(€)$ & Montant versé $(€)$ & \% réalisé \\
\hline SM3E & 760 & 296 & 39 \\
Usagers de l'électricité & 686 & 226 & 33 \\
Commune de Tolongoina & 686 & 267 & 39 \\
Total & 2132 & 789 & 37 \\
\hline
\end{tabular}

\section{Interprétation des attitudes des fournisseurs de services environnementaux}

Des considérations d'ordre économique, d'ordre social et cognitif, et d'ordre agraire apportent un éclairage dans la compréhension des attitudes des fournisseurs de services environnementaux.

\subsection{Facteurs de consentement à signer le contrat de PSE}

Les entretiens ont montré une adhésion des fournisseurs aux arguments socio-économiques avancés par l'équipe du GRET dans les différentes réunions pour la mise en place du PSE.

L'argument économique reposait principalement, d'une part, sur l'arbitrage entre contraintes induites par l'arrêté communal et possibilités, même limitées, offertes par le dispositif de PSE; d'autre part, sur l'espérance liée à des perspectives d'appuis plus conséquents à moyen et à court terme. Il vaut mieux avoir une compensation limitée au départ, mais d'une manière continue et qu'on espère croissante (accroissement prévu des usagers) dans le cadre du PSE, que de ne rien percevoir dans le cadre de l'arrêté communal et courir le risque d'un classement de certaines zones de culture du bassin versant en zone de protection liée à l'hydroélectricité (mise en défens, expulsions...).

$\mathrm{Au}$ vu de la situation de précarité induite par l'arrêté communal, les fournisseurs de services environnementaux ont considéré le montant de $1100 € /$ an prévu dans le contrat de PSE comme un «bonus». En effet, le contrat de PSE de 2013 n'a pas fondamentalement aggravé les règles de l'interdiction des feux de 2007, mais les a plutôt réaffirmées en les justifiant, et les a précisées. Dans un contexte tendu et incertain, à l'avenir risqué, on «accepte» ce qui est promis, même si la compensation au titre des services environnementaux est inférieure au montant espéré à l'issue du calcul des coûts d'opportunité.

Quant à l'argumentation sociale et cognitive, le GRET a présenté le dispositif de PSE comme source de reconnaissance des usagers des sols en amont. Vivant auparavant à la marge de la communauté et souvent accusés de la dégradation de l'environnement, ils sont désormais considérés comme des acteurs qui comptent, «utiles à la société». Le PSE offre à ces allochtones fournisseurs de services environnementaux (descendants de migrants récemment installés, Fig. 1) un outil d'intégration sociale auprès des autochtones.
Pour les fournisseurs de services environnementaux, la structuration de TAMIS et leur adhésion au sein du KOMSAHA améliorent leur capital social et permettent d'apaiser le climat social tendu entre les habitants situés en amont et ceux situés en aval.

Ces considérations ont amené les usagers des sols à accepter un niveau de compensation défavorable. Ils nous ont souvent dit dans les ateliers de « co-définition » : «On veut bien rendre service aux usagers de l'eau, on ne leur demande pas d'argent, mais on a besoin d'aide pour nous adapter aux règles de l'interdiction de $2007 »$. Ces considérations cadrent bien avec la notion de «services environnementaux ». Obtenir quelque chose, un contrat, avait aussi une valeur symbolique importante.

\subsection{Explication du respect des engagements}

Les entretiens effectués en 2016 ont montré que des dimensions sociales et cognitives ont également contribué au respect du contrat. Les paysans usagers des sols du bassin versant ont eu à cœur, durant la période du contrat, de montrer qu'ils ne constituaient pas un «danger» pour la société et méritaient «respect» et «reconnaissance» dans les rapports sociaux locaux. Le fait d'être mieux intégrés, d'une part à la société locale par leur participation à la plateforme KOMSAHA et par les multiples ateliers in situ en présence des officiels de Tolongoina, et d'autre part à la société nationale via les liens avec le GRET et les séjours des stagiaires, des chercheurs et d'évaluateurs étrangers, a compté pour eux.

Par ailleurs, des considérations agraires et économiques sont à prendre en compte pour comprendre l'observance des engagements des fournisseurs de services environnementaux.

Tout d'abord, des gains non monétaires, sous forme d'apprentissages techniques (agroforesterie et lutte antiérosive, co-construction de plans d'aménagement, gestion de contrats...), sont également pris en compte par les fournisseurs de services environnementaux, au-delà de la dotation de poulets par KOMSAHA en 2014, le seul paiement reçu dans le cadre du PSE.

Ensuite, une profonde transformation en cours des systèmes de production et une capacité d'adaptation des activités pratiquées (Ratianarijaona et al., 2016; Rakotoarisoa et al., 2016) ont facilité le respect des règles de l'arrêté communal de 2007 réactivées par le PSE de 2013.

En 2009, on comptait 30 ménages résidents $(120$ personnes environ) sur la zone agricole et 40 exploitations, soit une densité exploitante de l'ordre de $150 \mathrm{hab} . / \mathrm{km}^{2}$, montrant l'attractivité de ce site triplement propice à la banane (climat, 

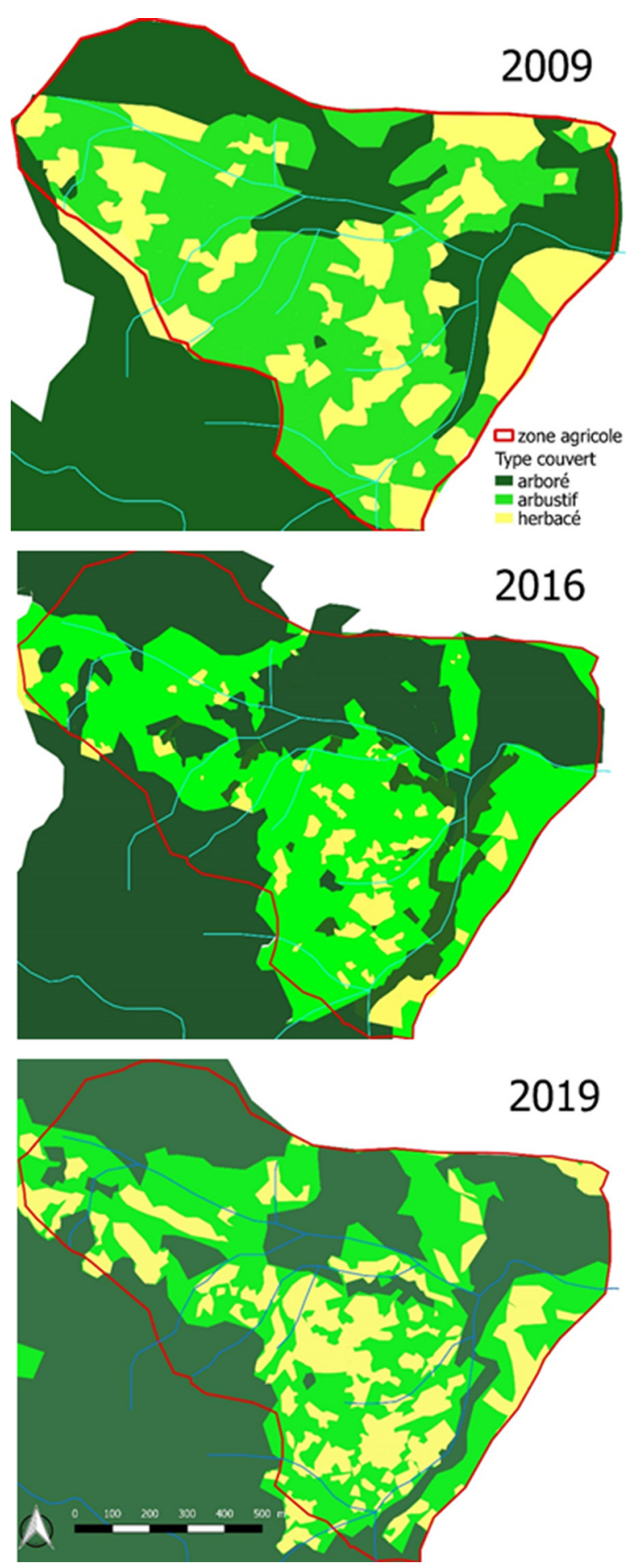

Fig. 4. Changement de physionomie d'occupation des terres entre 2009, 2016 et 2019 (Rakotoarisoa et al., 2016; auteurs).

Fig. 4. Land use change between 2009, 2016 and 2019.

sol forestier, amont d'une voie ferrée). Les systèmes de culture comprenaient des cultures vivrières: rares rizières, manioc, maïs-haricots sur jachère inférieure à 5 ans reprise par le feu. Des opérations de défriche-brûlis de forêt ont servi à étendre le domaine cultivé, mais il existait aussi des méthodes d'expansion plus vertueuses de type abattis-paillis (défriche sans feu, suivie de culture de bananiers). Les cultures de rente pérennes dominaient : banane, canne à sucre, café, fruitiers. Le gingembre en culture annuelle commençait çà et là. La moitié des ménages étaient des porteurs travaillant pour les planteurs. Bien que leurs droits à cultiver soient parfois mis en doute, certains usagers de la terre louaient leurs terres à des « investisseurs » de Tolongoina pour le gingembre.

En 2016, la situation a changé (Tab. 2). La population résidente est restée la même, mais beaucoup de ménages sont partis, et d'autres se sont installés. Le tableau 2 montre une augmentation du gingembre (le feu ne lui est pas nécessaire, en revanche le travail du sol est indispensable), une baisse de la banane, remplacée par la canne à sucre et le toaka gasy, un rhum artisanal illicite mais lucratif. Les élevages de porcs ont augmenté, ainsi que les terres hors bassin versant (rizières, champs de haricot). Le salariat a beaucoup diminué avec la réduction de la banane, qui n'est plus la première source de revenu.

L'évolution du système de production, conforme au cahier des charges du PSE, s'explique ici par cette capacité de la communauté rurale à se réadapter (renonciation au feu, maintien de la population exploitante au niveau de 2009, adaptations en cours basées sur les plantations pérennes, diversification par l'élevage, et mobilité [déplacement d'exploitations et d'activités vivrières hors bassin versant]), éventuellement au détriment d'autres forêts, parfois au profit d'activités illicites.

Après 2013, des nouveaux venus, conscients des règlements de gestion du bassin versant, les ont respectés durant le contrat. Certains sont partis en poursuivant leurs pratiques habituelles ailleurs. Dans les deux cas, les engagements des usagers du bassin versant dans le contrat de PSE ont toujours été respectés. Les usagers du bassin versant étaient conscients que l'engagement dans le contrat constituait la raison de l'abandon de certaines pratiques. La situation observée en 2019 s'explique par la fin du contrat, qui signifie également rupture des engagements, situation aggravée par le problème socio-organisationnel et de gouvernance dans le comité de bassin KOMSAHA.

\section{Discussion et conclusion}

Dans un contexte difficile, marqué notamment par une grande pauvreté et une précarité foncière, le dispositif de PSE a été considéré par les usagers des sols, devenus fournisseurs de services environnementaux, comme: (i) une opportunité en matière de financement collectif; (ii) une reconnaissance sociale; et (iii) un renforcement de leur position en matière de statut d'occupation foncière, auparavant marqué par des poursuites en cas de défrichement, des menaces et des tracasseries administratives. Ces différentes considérations viennent «au secours» des incitations économiques directes insuffisantes de ce PSE et compensent la faiblesse de la compensation prévue et effective ainsi que la mauvaise application du contrat.

Nous retrouvons ici, d'une part, des motivations d'ordre intrinsèque, qui se définissent comme «une motivation autodéterminée ou autonome, inspirée notamment par des convictions personnelles ou des défis, ou relative à une activité qui procure en elle-même de la satisfaction » (Deci et Ryan, 
Tableau 2. Evolution du système d'activités des ménages usagers du bassin versant. Table 2. Evolution of the activity systems of the user households of the watershed.

\begin{tabular}{lcc}
\hline & 2009 & 2016 \\
\hline Nombre de ménages usagers du bassin versant & 40 & 43 \\
Nombre de ménagés interrogés & 30 & 39 \\
Activités & & 39 \\
Banane & 63 & 26 \\
Canne à sucre et toaka gasy & 0 & 70 \\
Gingembre & 33 & 51 \\
Terres exploitées en dehors du bassin versant & 40 & 70 \\
Élevage de porcs & 53 & 8 \\
Activités salariées & 53 & \\
\hline
\end{tabular}

2000); et d'autre part, des motivations d'ordre extrinsèque, qui «relèvent d'incitations extérieures liées à un effet carotte (rémunération, promesses...) ou à un effet bâton (surveillance, menace, sanction) » (Merlateaux, 2011).

Dans le domaine des PSE, les observations effectuées par Rode et al. (2015) ou Akers et Yasué (2019) montrent qu'une partie de la littérature sur les PSE, s'appuyant notamment sur des recherches en psychologie, a voulu opposer les motivations intrinsèques et extrinsèques, où l'une des deux peut annuler, inhiber ou évincer l'autre. Cet article nous montre au contraire un enchevêtrement de ces deux catégories de motivations. La combinaison de facteurs économiques, sociaux et institutionnels explique les motivations des fournisseurs de services environnementaux et leur propension à s'engager dans ce PSE et à honorer leur propre engagement jusqu'à l'échéance du contrat qui les en libérait.

Ces résultats mettent en exergue une complémentarité de facteurs économiques ou hybrides; ils sont cohérents avec d'autres recherches relatives au niveau d'engagement dans les différentes étapes d'un PSE et les motivations afférentes. Nous citerons notamment le renforcement des motivations sociales par le système de paiements collectifs (Moros et al., 2019); l'importance de facteurs économiques complémentaires comme les caractéristiques des ménages et de la communauté (Adhikari et Agrawal, 2013); l'acceptation de rémunérations inférieures au coût d'opportunité par des communautés rurales pauvres confrontées d'une manière récurrente aux variations du marché et aux aléas climatiques (Wunder, 2005); ainsi que la prise en compte de facteurs non économiques et cognitifs allant au-delà de la pure rationalité économique (Kosoy et al., 2008; Lapeyre et al., 2016).

Le comportement des fournisseurs de services environnementaux situe ce PSE de Tolongoina dans une zone intermédiaire entre vrai PSE de nature exclusivement économique (Ferraro et Kiss, 2002; Wunder, 2005) et dispositifs réglementaires avec mesures de répression allégées et outils de sensibilisation - un cadre contractuel « mou» et une «carotte» symbolique. Il a une dimension éminemment sociale, au-delà de toute logique monétaire.

Cet article montre qu'un PSE n'a rien de mécanique et soulève également d'autres pistes de recherche comme la problématique de l'action désintéressée de conservation de la nature et des conséquences de la nécessité d'être payé pour respecter les lois (Karsenty et al., 2017) ou celle relative à la mise en perspective des coûts de mise en place ou de mise en œuvre pour un tel PSE, par rapport aux compensations prévues et effectivement perçues par les fournisseurs de services environnementaux. En matière d'implications opérationnelles et de construction de politiques, les éléments mis en évidence pourraient intéresser les décideurs publics, les bailleurs de fonds et les autres praticiens.

\section{Références}

Adhikari B, Agrawal A. 2013. Understanding the Social and Ecological Outcomes of PES Projects: a review and an analysis. Conservation \& Society 11(4): 359-374. DOI: 10.4103/ 0972-4923.125748.

Akers JF, Yasué M. 2019. Motivational Crowding in Payments for Ecosystem Service Schemes: a Global Systematic Review. Conservation and Society 17(4): 377-389. DOI: 10.4103/cs. cs 1890 .

Andriamahefazafy F, Fetiveau J, Lapeyre R. 2017. Analyse du PSE de Tolongoina: quels enseignements pour les futurs PSE à Madagascar? Antananarivo: GRET, $38 \mathrm{p}$.

Bidaud C, Méral P, Andriamahefazafy F, Serpantié G, Cahen-Fourrot L, Toillier A. 2013. Institutional and Historical Analysis of Payments for Ecosystem Services in Madagascar. In: Muradian R, Rival L, eds. Governing the Provision of Ecosystem ServicesStudies in Ecological Economics, Vol.4, pp. 207-232. Dordrecht: Springer. DOI: 10.1007/978-94-007-5176-7_11.

Bremer LL, Farley KA, Lopez-Carr D. 2014. What factors influence participation in payment for ecosystem services programs? An evaluation of Ecuador's SocioPáramo program. Land Use Policy 36: 122-133. DOI: 10.1016/j.landusepol.2013.08.002.

Deci EL, Ryan RM. 2000. Intrinsic and Extrinsic Motivations: Classic Definitions and New Directions. Contemporary Educational Psychology 25(1): 54-67. DOI: 10.1006/ceps.1999.1020.

Ferraro PJ, Kiss A. 2002. Direct Payments to Conserve Biodiversity. Science 298 (5599): 1718-1719. DOI: 10.3126/hjs.v1i2.200.

Froger G, Méral PH, Muradian R. 2016. Vers une prise en compte de la diversité des arrangements institutionnels et des pratiques dans l'analyse des paiements pour services environnementaux. Développement durable et territoires [En ligne] 7(1). http://developpe mentdurable.revues.org/11163.

Karsenty A. 2019. Les paiements pour services environnementaux dans les pays en développement: compenser ou récompenser? In : Langlais A, ed. L'agriculture et les paiements pour services environnementaux: quels questionnements juridiques? Rennes: Presses universitaires de Rennes, pp. 79-100. 
Karsenty A, Aubert S, Brimont L, Dutilly C, Desbureaux S, Ezzine de Blas E, et al. 2017. The Economic and Legal Sides of Additionality in Payments for Environmental Services. Environmental Policy and Governance 27(5): 422-435. DOI: 10.1002/eet.1770.

Kosoy N, Corbera E, Brown K. 2008. Participation in payments for ecosystem services: Case studies from the Lacandon rainforest, Mexico. Geoforum 39(6): 2073-2083. DOI: 10.1016/j.geofo rum.2008.08.007.

Lapeyre R, Pirard R, Leimona B. 2016. Paiements pour services environnementaux en Indonésie: incitations économiques ou motivations sociales? Développement durable et territoires [En ligne] 7(1). http://journals.openedition.org/developpementdurable/ 11147.

Méral P. 2012. Le concept de service écosystémique en économie : origine et tendances récentes. Nature Sciences et Sociétés 20(1): 30-38. DOI: $10.1051 / \mathrm{nss} / 2012002$.

Merlateaux M. 2011. Incitations monétaires et motivation au travail : de la théorie économique à la neuroéconomie. Économie et institutions 16. DOI: 10.4000/ei.78.

Moros L, Vélez MA, Corbera E. 2019. Payments for Ecosystem Services and Motivational Crowding in Colombia's Amazon Piedmont. Ecological Economics 156(C): 468-488. DOI: 10.1016/ j.ecolecon.2017.11.032.

Moyen C. 2010. Étude d'accompagnement à la mise en place d'un dispositif de «Paiement pour Services Environnementaux» de régulation hydrologique dans la commune de Tolongoina (Madagascar). Mémoire de fin d'études d'ingénieur, spécialité gestion sociale de l'eau. Montpellier: ENGEES/IRC-SUPAGRO, $90 \mathrm{p}$.

Rakotoarisoa L, Vogel A, Serpantié G. 2016. État des lieux du dispositif de PSE Tolongoina en fin de contrat: volet agricole et environnemental. Rapport de consultance. Montpellier: GRET/ IRD/C3EDM, $36 \mathrm{p}$.

Rakotonirina A. 2018. Les Paiements pour Services Environnementaux (PSE), comme nouvel outil de gestion des bassins versants de sites hydroélectriques: cas des microcentrales à Madagascar. In: Communication aux $34^{e}$ journées du développement de l'Association Tiers Monde, Université Grenoble Alpes.

Ratianarijaona M, Vogel A, Serpantié G, Andriamahefazafy F. 2016. Le PSE Tolongoina : état des lieux en fin de contrat. Volet socioéconomique - Rapport de consultance. Antananarivo : GRET-IRDC3EDM, $52 \mathrm{p}$.

Roche C. 2011. Construction d'une démarche de co-définition du Service Environnemental dans le cadre de la mise en œuvre d'un Paiement pour Service Environnemental. Exemple du bassin versant d'Andasy. Mémoire de fin d'Etudes. Cergy-Pontoise. Montpellier: ISTOM, IRD, $116 \mathrm{p}$.

Rode J, Baggethun EG, Krause T. 2015. Motivation crowding by economic incentives in conservation policy: A review of the empirical evidence. Ecological Economics 117(C): 270-282. DOI: 10.1016/j.ecolecon.2014.11.019.

Shapiro-Garza E, McElwee P, Van Hecken G, Corbera E. 2020. Beyond Market Logics: Payments for Ecosystem Services as Alternative Development Practices in the Global South. Development and Change 51(1): 3-25. DOI: 10.1111/dech.12546.

Toillier A. 2009. Etudes des possibilités de mise en œuvre d'un PSE pour la protection du bassin versant de la microcentrale hydroélectrique de Tolongoina-Madagascar. Antananarivo: GRET, $150 \mathrm{p}$.

Wunder S. 2005. Payments for Environmental Services: Some Nuts and Bolts. CIFOR Occasional Paper 42. Bogor, Indonesia: Center for International Forestry Research.

Citation de l'article : Andriamahefazafy F, Rakotonirina A, Serpantié G. 2020. Paiement pour services environnementaux (PSE) et motivations des usagers des terres d'un bassin versant. Cas de l'hydroélectricité à Tolongoina, Madagascar. Cah. Agric. 29: 36. 